University of Nebraska - Lincoln

DigitalCommons@University of Nebraska - Lincoln

Robert Streubel Papers

Research Papers in Physics and Astronomy

$1-25-2016$

\title{
Vortex circulation and polarity patterns in closely packed cap arrays
}

Robert Streubel

Leibniz-Institut für Festkörper- und Werkstoffforschung Dresden, streubel@unl.edu

Florian Kronast

Helmholtz-Zentrum Berlin für Materialien und Energie (HZB)

Christopher F. Reiche

Leibniz-Institut für Festkörper- und Werkstoffforschung Dresden

Thomas Mühl

Leibniz-Institut für Festkörper- und Werkstoffforschung Dresden

Anja U.B. Wolter

Leibniz-Institut für Festkörper- und Werkstoffforschung Dresden

See next page for additional authors

Follow this and additional works at: https://digitalcommons.unl.edu/physicsstreubel

Part of the Atomic, Molecular and Optical Physics Commons, Condensed Matter Physics Commons, and the Other Physics Commons

Streubel, Robert; Kronast, Florian; Reiche, Christopher F.; Mühl, Thomas; Wolter, Anja U.B.; Schmidt, Oliver G.; and Makarov, Denys, "Vortex circulation and polarity patterns in closely packed cap arrays" (2016). Robert Streubel Papers. 16.

https://digitalcommons.unl.edu/physicsstreubel/16

This Article is brought to you for free and open access by the Research Papers in Physics and Astronomy at DigitalCommons@University of Nebraska - Lincoln. It has been accepted for inclusion in Robert Streubel Papers by an authorized administrator of DigitalCommons@University of Nebraska - Lincoln. 


\section{Authors}

Robert Streubel, Florian Kronast, Christopher F. Reiche, Thomas Mühl, Anja U.B. Wolter, Oliver G. Schmidt, and Denys Makarov 


\section{Vortex circulation and polarity patterns in closely packed cap arrays}

Cite as: Appl. Phys. Lett. 108, 042407 (2016); https://doi.org/10.1063/1.4941045

Submitted: 09 November 2015 • Accepted: 19 January 2016 • Published Online: 28 January 2016

Robert Streubel, Florian Kronast, Christopher F. Reiche, et al.
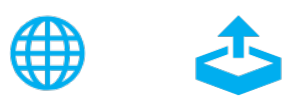

View Online

\section{ARTICLES YOU MAY BE INTERESTED IN}

Shapeable magnetoelectronics

Applied Physics Reviews 3, 011101 (2016); https://doi.org/10.1063/1.4938497

Equilibrium magnetic states in individual hemispherical permalloy caps

Applied Physics Letters 101, 132419 (2012); https://doi.org/10.1063/1.4756708

Magnetic anisotropy engineering: Single-crystalline Fe films on ion eroded ripple surfaces Applied Physics Letters 100, 242405 (2012); https://doi.org/10.1063/1.4729151

\section{Challenge us.}

What are your needs for periodic signal detection?

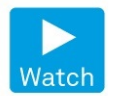

Zurich

Instruments

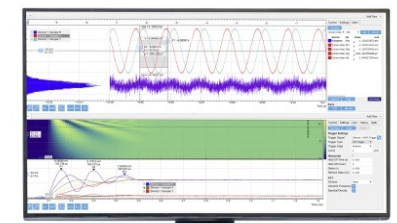

( 


\title{
Vortex circulation and polarity patterns in closely packed cap arrays
}

\author{
Robert Streubel, ${ }^{1, \text { a) }}$ Florian Kronast, ${ }^{2}$ Christopher F. Reiche, ${ }^{3}$ Thomas Mühl, $^{3}$ \\ Anja U. B. Wolter, ${ }^{3}$ Oliver G. Schmidt, ${ }^{1,4}$ and Denys Makarov ${ }^{1, b)}$ \\ ${ }^{1}$ Institute for Integrative Nanosciences, IFW Dresden, 01069 Dresden, Germany \\ ${ }^{2}$ Helmholtz-Zentrum Berlin für Materialien und Energie GmbH, 12489 Berlin, Germany \\ ${ }^{3}$ Institute for Solid State Research, IFW Dresden, 01069 Dresden, Germany \\ ${ }^{4}$ Material Systems for Nanoelectronics, Chemnitz University of Technology, 09107 Chemnitz, Germany
}

(Received 9 November 2015; accepted 19 January 2016; published online 28 January 2016)

\begin{abstract}
We studied curvature-driven modifications to the magnetostatic coupling of vortex circulation and polarity in soft-magnetic closely packed cap arrays. A phase diagram for the magnetic remanent/ transition states at room temperature as a function of diameter and thickness was assembled. For specimens with vortex remanent state ( $40 \mathrm{~nm}$-thick Permalloy on $330 \mathrm{~nm}$ spherical nanoparticles), both vortex circulation and polarity were visualized. Intercap coupling upon vortex nucleation leads to the formation of vortex circulation patterns in closely packed arrays. The remanent circulation pattern can be tailored choosing the direction of the applied magnetic field with respect to the symmetry axis of the hexagonal array. An even and random distribution of vortex polarity indicates the absence of any circulation-polarity coupling. ( 2016 AIP Publishing LLC.

[http://dx.doi.org/10.1063/1.4941045]
\end{abstract}

Curvature-driven effects ${ }^{1-3}$ were proposed theoretically as an efficient way to manipulate the response of three-dimensionally (3D) shaped magnetic objects. Main research activities focus on topological patterning, ${ }^{4-6}$ nonlocal interaction, ${ }^{6}$ and chirality symmetry breaking ${ }^{1,2,7-10}$ of the magnetization. These physical mechanisms manifest themselves in unidirectionality of magnon spin excitations (magnon Cherenkov effect), ${ }^{11}$ unlimited domain wall velocities ${ }^{12}$ and geometrically induced skyrmionic spin textures. ${ }^{6}$ One of the puzzling predictions is an emergent curvature-driven Dzyaloshinskii-Moriya-like interaction (DM-like interaction) $)^{3,5,13}$ in curved/bent surfaces originating from an intrinsic space inversion that adds up to the Heisenberg spin exchange.

Providing evidence of these theoretical predictions requires high-quality magnetic thin films with structural symmetry to avoid competing magnetostatic contributions owing to demagnetization fields and dipole-dipole interaction. An elegant way is to deposit magnetic films onto curvature templates with various shape and structural arrangement ${ }^{14-18}$ allowing for tuning exchange and magnetostatic interactions at the nanoscale. ${ }^{19-21}$ By these means, it was demonstrated that magnetic vortex states can be stabilized in closely packed soft-magnetic cap structures ${ }^{16-18,22-25}$ and reconfigured in thickness-modulated Honeycomb lattices. ${ }^{26}$ Although a negligibly small stray field exists at remanence, the strong magnetostatic dipolar interaction during vortex nucleation could favor peculiar macroscopic magnetic patterns on closely packed vortex lattice. ${ }^{16}$ The deterministic vortex circulation arrangement combined with an analysis of the vortex core profile for different polarities provide means to address the

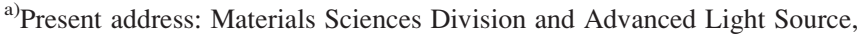
Lawrence Berkeley National Laboratory, Berkeley, California 94720, USA. Electronic mail: streubel@lbl.gov

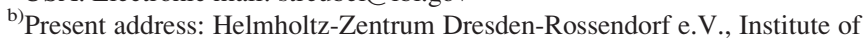
Ion Beam Physics and Materials Research, 01328 Dresden, Germany. Electronic mail: d.makarov@hzdr.de
}

question about the presence of curvature-driven DM-like interaction in polycrystalline cap structures, which would manifest itself in a chirality-dependent extension of the vortex core. $^{27}$

Here, we report the frustration-driven formation of vortex circulation patterns in arrays of Permalloy $\left(\mathrm{Py}, \mathrm{Ni}_{80} \mathrm{Fe}_{20}\right)$ cap structures visualized by X-ray magnetic circular dichroism (XMCD) photoemission electron microscopy (XPEEM). A full parameter range phase diagram of magnetic remanent and equilibrium states in closely packed cap arrays is given as a function of film thickness and cap diameter. We focus our discussion on arrays consisting of $40 \mathrm{~nm}$-thick Permalloy caps on spheres with a diameter of $330 \mathrm{~nm}$ that show a coupled vortex circulation pattern at remanence and room temperature. The circulation patterns are analyzed at remanence after saturating the sample along different directions with respect to the symmetry axes of the hexagonal cap array. Joint XPEEM and high-resolution magnetic force microscopy (HR-MFM) studies reveal the absence of coupling between vortex circulation and polarity and thus of a Dzyaloshinskii-Moriya interaction (DMI $)^{28,29}$ or DM-like interaction in our polycrystalline samples-in contrast to the report by Im et al. ${ }^{30}$ In this respect, the magnetic cap structures behave differently compared to the thin spherical shells. ${ }^{6}$

The curvature template is obtained by drop-casting silica particles onto a Si wafer. This way, monolayers consisting of spheres with a diameter in the range of $\varnothing=(50-800) \mathrm{nm}$ are prepared, which are capped by a $\mathrm{Ta}(5 \mathrm{~nm}) / \mathrm{Py}(t) / \mathrm{Ta}(2 \mathrm{~nm})$ layer stack. The thickness of the Py film $t$ was varied from 10 to $40 \mathrm{~nm}$. The metallic layers are deposited via dc magnetron sputtering at room temperature (base pressure: $7 \times 10^{-8} \mathrm{mbar}$; Ar pressure: $10^{-3} \mathrm{mbar}$, and rate: $1 \AA / \mathrm{s}$ ). Directed deposition is ensured by positioning an aperture right above the substrate generating a curvature-driven thickness gradient with the nominal thickness $t$ at the pole and a persistently decreasing thickness when approaching the 
equator [Fig. 1(a)]. It has already been shown that such a system stabilizes the vortex state as schematized in Fig. 1(a) even in closely packed cap arrays, when Py film thickness and particle diameter are adjusted properly. ${ }^{18}$

Studying samples with different film thickness and sphere diameter, the impact of the structural geometry, e.g., curvature, on coupling and magnetization patterns is investigated. The magnetic hysteresis loops are recorded utilizing superconducting quantum interference device (SQUID) magnetometry at room temperature. The shape of the hysteresis loops varies for samples with different Py thickness and sphere diameter as exemplarily shown in Fig. 1(b). In particular, samples with a Py film thickness $t=40 \mathrm{~nm}$ and diameters of $\varnothing=330 \mathrm{~nm}$ and larger reveal a hysteresis loop typically observed for confined magnetic nanoobjects in a magnetic vortex state ${ }^{31}$ with characteristic nucleation and annihilation fields. ${ }^{16}$ The nucleation field refers to the magnetic field with the largest susceptibility at which the vortex core is nucleated [Fig. 1(b), $\left|m_{s}\right| \approx 0.5$ ]. Evidence of this assumption is given below by directly visualizing the in-plane magnetization components and the perpendicular magnetostatic force gradient using XPEEM and HR-MFM, respectively. Smaller cap radii suppress a vortex nucleation promoting a rotation of the magnetization in the onion state in the cap. ${ }^{16}$ On the other hand, the vortex state becomes less stable, manifested by smaller annihilation and nucleation fields, with decreasing Py thickness while keeping the diameter unchanged and is finally suppressed completely.
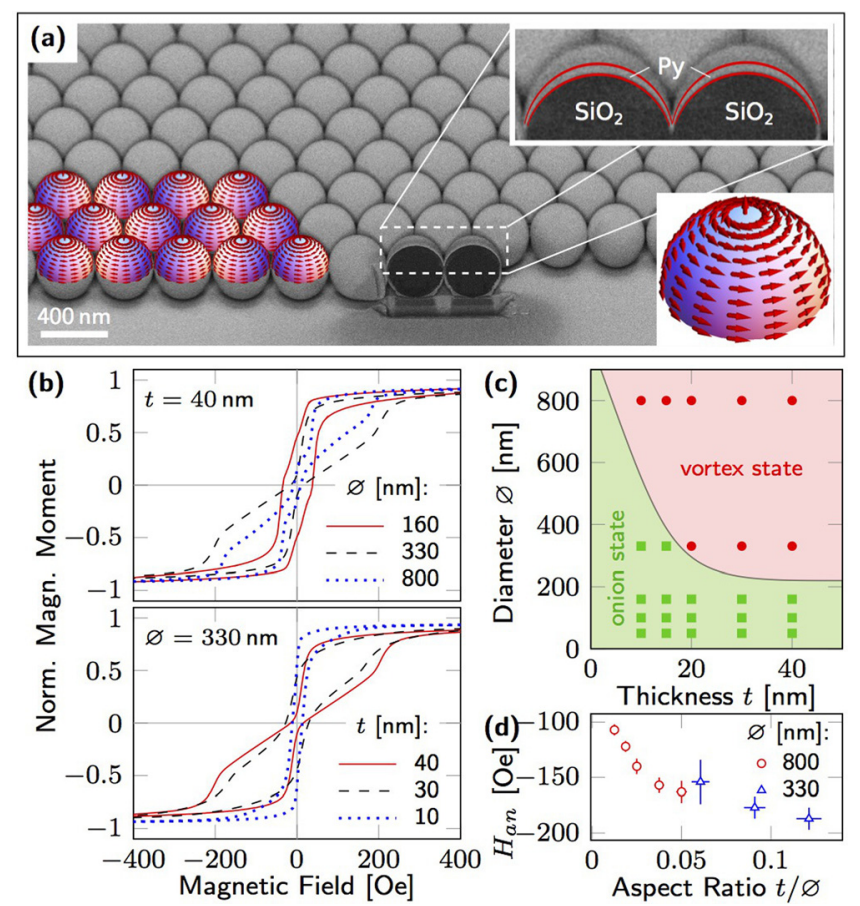

FIG. 1. (a) Soft-magnetic Py caps on $\mathrm{SiO}_{2}$ particles that exhibit a thickness gradient stabilizing the vortex state even in closely packed arrays. (b) Magnetic hysteresis curves for Py films deposited onto spheres of various diameters and various thicknesses recorded with SQUID magnetometry at room temperature while sweeping the magnetic field perpendicularly to the substrate surface. (c) Magnetic phase diagram at room temperature. The two magnetic phases, vortex and onion state, are indicated with a solid line as a guide-to-the-eye. (d) Vortex annihilation field as a function of the aspect ratio.
The accordingly derived phase diagram of magnetic states for the whole parameter range is depicted in Fig. 1(c). The solid line separating the two phases, namely, vortex and onion state, is given as a guide-to-the-eye and represents the phase boundary. The location of the phase boundary can be understood by the decrease in the aspect ratio, the quotient of film thickness, and diameter. ${ }^{32}$ For small aspect ratios (thin films), magnetic volume charges, and thus stray field contributions, become small, which represent the main driving force for stabilizing the vortex state. This simple argument does not hold for samples with small diameters $(\varnothing \lesssim 200 \mathrm{~nm}$, separation between vortex cores) since the magnetostatic interaction between adjacent caps increases significantly as known from planar disk arrays. ${ }^{32}$ Moreover, a large film thickness and small diameters $(\varnothing \leqq 50 \mathrm{~nm})$ lead to a modulation of an extended film instead of closely packed cap arrays. Comparing the experimental phase diagram for closely packed arrays with the numerically determined one for single caps ${ }^{18}$ reveals further an extended onion phase due to magnetostatic interaction during the vortex nucleation process. Contrary to their planar counterparts, ${ }^{32}$ vortices in closely packed cap arrays exhibit vortex annihilation fields that do not only depend on the aspect ratio $t / \varnothing$, but also on the cap diameter $\varnothing$ [Fig. 1(d)]. The occurrence of two regions with distinct slope cannot only be assigned to an altered magnetostatic interaction owing to an

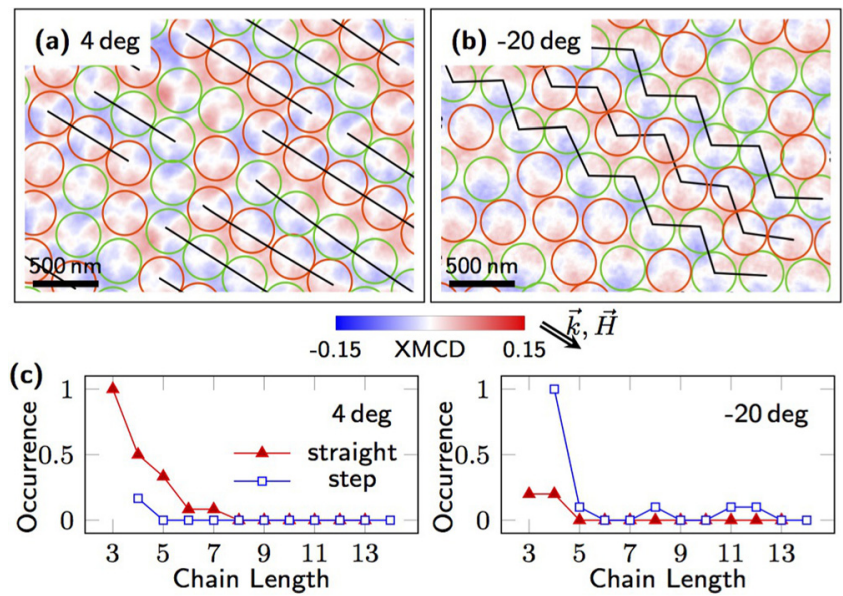

(d) Periodic arrangements for circulation
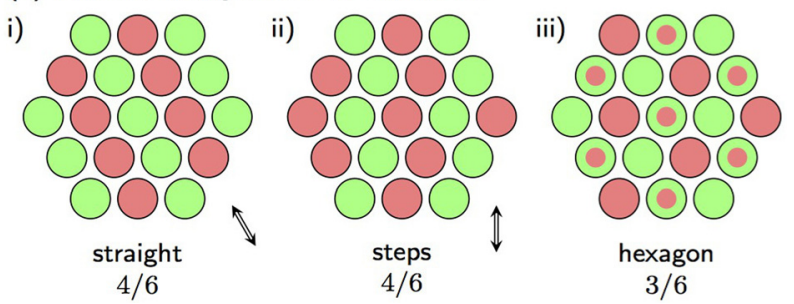

FIG. 2. Vortex circulation patterns in closely packed cap arrays. Remanent states for two different cap arrays initially saturated in a magnetic field (400 Oe) oriented (a) $4^{\circ}$ and (b) $-20^{\circ}$ with respect to the magnetic field. The vortex circulation is indicated by red and green circles. (c) Histogram of straight lines and steps that form the patterns shown in (d) to calculate the lateral expansion of the domains. (d) Possible periodic arrangement of circulation-coupled vortices in hexagonal arrays. Red and green circles indicated vortex circulation; Green-red circles refer to caps with no specific circulation. The original saturation direction is indicated by arrows. Straight lines and steps represent the lowest ground state with 4 of 6 nearest neighbors matching alternating circulation. Arrangements with a three-fold symmetry are less preferable in contrast to the case of Kagomé lattices due to the frustration of the green-red circles. 
effectively increased intercap separation for larger spheres, which feature a smooth transition, ${ }^{32}$ but also to the curvature itself.

The interpretation of the magnetic hysteresis loops suggests the vortex nucleation in spin exchange decoupled nanoscopic caps $(\varnothing \gtrsim 200 \mathrm{~nm})$. Utilizing XPEEM, the in-plane magnetization components and particularly the vortex circulation are visualized providing insight into magnetostatic interaction on the magnetic pattern within closely packed cap arrays $(t=40 \mathrm{~nm}, \varnothing=330 \mathrm{~nm})$. Figures 2(a) and 2(b) depict the remanent states for two different cap arrays initially saturated in a magnetic field $(400 \mathrm{Oe})$ oriented $4^{\circ}$ and $-20^{\circ}$ with respect to the main symmetry axis of the hexagonal array. The field was ramped down to zero within a few seconds. The magnetization within each cap forms the typical quadrupole XMCD contrast of vortices in thick films $(t \gtrsim 20 \mathrm{~nm}) .{ }^{18}$ The vortex circulation is indicated by green and red circles. The array initially saturated close to the symmetry axis $\left(4^{\circ}\right.$; main symmetry axis is along $\left.0^{\circ}\right)$ reveals straight chains of same circulation, which alternate perpendicular to the $0^{\circ}$ orientation. If the sample is saturated along a direction close to the second symmetry axis of the array $\left(-20^{\circ}\right.$; second symmetry axis is along $\left.-30^{\circ}\right)$, steps assemble that alternate in the same way. A quantitative analysis is given in Fig. 2(c) in form of histograms for patterns consisting of straight lines and steps. The vortex circulation patterns are extended over several caps. A stochastic distribution favoring such an arrangement is very unlikely. In order to stabilize such a pattern, an interaction, i.e., magnetostatic coupling, has to be present during the vortex nucleation process. Considering magnetostatic interaction between nearest neighboring caps during vortex nucleation, both arrangements represent the ground state with four nearest neighbors having opposite circulation for the corresponding array orientation [Fig. 2(d)]. Although sometimes observed, the three-fold arrangement is less favored because merely half of nearest neighbors match circulation.

However, the considerations about ground states only apply for collective transitions, since the system is not frustrated at remanence due to vanishing magnetostatic interaction between adjacent vortices. The term "collective" refers to the fact that the circulation of each vortex is predetermined by the magnetostatic nearest neighbor interaction in the array during the vortex nucleation process. In particular, the local magnetization during the magnetization reversal process is affected by the lattice orientation of the closely packed cap array and forms meander-like flux-closure domains with nodes located at the intersect of adjacent caps. These modulations, eventually leading to the circulation patterns, possess a certain coherence that is described by the lateral expansion of the circulation patterns in units of caps [Fig. 2(c)]. The lateral expansion of the vortex circulation patterns depends on the interplay between magnetostatic interaction and thermal fluctuations. It is defined as the (integer) number of caps where the normalized occurrence of straight lines and steps of caps with the same circulation is $1 / \mathrm{e}$. For an array exposed to the field aligned $4^{\circ}$ with respect to the main symmetry axis, the lateral expansion at room temperature is four caps [Fig. 2(c)]. 57\% of all caps are surrounded by four caps with matching circulation; $25 \%$ have three nearest neighbors with opposite circulation, independently of the crystal orientation. This indicates that roughly $40 \%$ of all caps are located at the grain boundaries. The coupling strength is too small to ensure a long-range arrangement of circulation at room temperature due to thermal fluctuations. A similar trend is observed for steps and a sample exposed to the magnetic field applied along $-20^{\circ}$. Additionally, step formations ranging over more than 10 caps were observed [Fig. 2(c)], hinting for a more efficient coupling mechanism. Indeed, micromagnetic simulations for crystal orientations close to $30^{\circ}$ reveal that vortices of adjacent caps nucleate pairwise close to each other and experience a much larger dipole-dipole interaction compared to the case of $0^{\circ}$ orientation.

The second property of the magnetic vortex state, namely, polarity, is probed by frequency-modulation HRMFM. In order to remove contact potential force contributions, a bias voltage of $300 \mathrm{mV}$ is applied during measurement. Figure 3(a) displays a typical MFM contrast of closely packed vortices $(t=40 \mathrm{~nm}, \varnothing=330 \mathrm{~nm})$ with bright and dark regions corresponding to the vortex core in the center of the cap, which are indicated by dashed white circle. Positively or negatively charged core surfaces are distributed evenly in a random manner [Fig. 3(a)] in spite of the circulation patterns formation [Figs. 2(a) and 2(b)], which is in agreement with the recent report by Nissen $e t$ al. ${ }^{25}$ The profiles of positive (bright) and negative (dark) polarity are shown in Fig. 3(b). After subtracting electrostatic contributions using a Gaussian fit, all positive and negative profiles reveal the same shape with a FWHM of $(46 \pm 2) \mathrm{nm}$, which is broadened compared to the typical vortex core size of about $10 \mathrm{~nm}$ due to the convolution with the size of the MFM tip. The lack of a clear dependence of the vortex core size on the product of polarity and circulation ${ }^{27}$ may be caused by a weakened or absent DM-like interaction induced by curvature, surface, or interface due to: film thicknesses much larger than the exchange length; too small curvature; or a partial microscopic compensation in polycrystalline films. In order to separate these contributions, smaller cap structures with vortex state would be needed. Unfortunately, no vortex state is stabilized in thin caps with small (large) diameter (curvature), neither in closely packed [Fig. 1(c)] nor in individual caps. ${ }^{18}$ However, correlating vortex circulation
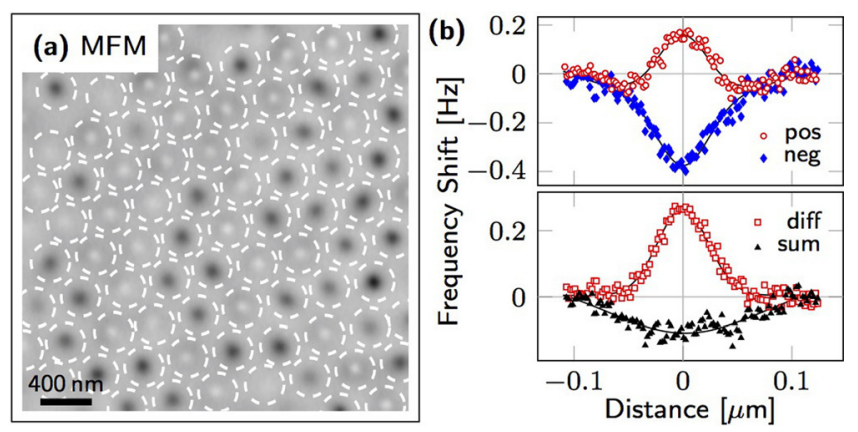

FIG. 3. (a) Magnetic force micrograph shows vortices with randomly distributed polarity (white and black contrast) localized at the center of the cap (indicated by white dashed circle) independent from the initial saturation orientation. (b) Line profiles for positive and negative polarity contain residual electrostatic contributions that are eliminated by subtraction, since profiles do not differ within the noise level. 
and polarity patterns with each other allows to extract contributions caused by the film thickness, as both vortex properties are set during the vortex nucleation process at the edge of the cap with a thickness comparable to the exchange length.

In conclusion, we prepared closely packed Permalloy cap arrays with various film thicknesses and cap diameters to assemble the magnetic phase diagram. Curvature-driven modifications lead to an extension of the vortex state phase towards smaller dimensions with respect to its planar counterpart. Whereas vortex polarity is evenly and randomly distributed, the hexagonal arrangement of caps induces a vortex circulation coupling. Depending on the initial saturation magnetization, straight lines or steps form demonstrating a frustration during vortex nucleation. For our geometry and samples, we further exclude both curvature-driven DM-like interaction and the hypothetically stated DMI reported for planar disks. $^{30}$

The authors thank I. Fiering (IFW Dresden) for sputter deposition, S. Gaß (IFW Dresden) for technical support of the SQUID-VSM, and Dr. V. Kravchuk (BITP Kyiv, Ukraine) and Dr. U. K. Rößler (IFW Dresden) for fruitful discussions. This work was financed via the European Research Council under European Union's Seventh Framework program (FP7/ 2007-2013)/ERC Grant Agreement No. 306277 and the Future and Emerging Technologies (FET) programme under FET-Open Grant No. 618083.

${ }^{1}$ P. Landeros and A. S. Núñez, J. Appl. Phys. 108, 033917 (2010).

${ }^{2}$ R. Hertel, SPIN 03, 1340009 (2013).

${ }^{3}$ Y. Gaididei, V. P. Kravchuk, and D. D. Sheka, Phys. Rev. Lett. 112, 257203 (2014).

${ }^{4}$ E. J. Smith, D. Makarov, S. Sanchez, V. M. Fomin, and O. G. Schmidt, Phys. Rev. Lett. 107, 097204 (2011).

${ }^{5}$ O. V. Pylypovskyi, V. P. Kravchuk, D. D. Sheka, D. Makarov, O. G. Schmidt, and Y. Gaididei, Phys. Rev. Lett. 114, 197204 (2015).

${ }^{6}$ V. P. Kravchuk, D. D. Sheka, R. Streubel, D. Makarov, O. G. Schmidt, and Y. Gaididei, Phys. Rev. B 85, 144433 (2012).

${ }^{7}$ M. Yan, C. Andreas, A. Kákay, F. García-Sánchez, and R. Hertel, Appl. Phys. Lett. 99, 122505 (2011).

${ }^{8}$ M. Yan, C. Andreas, A. Kákay, F. García-Sánchez, and R. Hertel, Appl. Phys. Lett. 100, 252401 (2012).
${ }^{9}$ J. A. Otalora, J. A. Lopez-Lopez, P. Vargas, and P. Landeros, Appl. Phys. Lett. 100, 072407 (2012).

${ }^{10}$ K. V. Yershov, V. P. Kravchuk, D. D. Sheka, and Y. Gaididei, J. Appl. Phys. 117, 083908 (2015).

${ }^{11}$ M. Yan, A. Kákay, C. Andreas, and R. Hertel, Phys. Rev. B 88, 220412 (2013).

${ }^{12}$ M. Yan, A. Kákay, S. Gliga, and R. Hertel, Phys. Rev. Lett. 104, 057201 (2010).

${ }^{13}$ D. D. Sheka, V. P. Kravchuk, and Y. Gaididei, J. Phys. A: Math. Theor. 48, 125202 (2015).

${ }^{14}$ M. Albrecht, G. Hu, I. L. Guhr, T. C. Ulbrich, J. Boneberg, P. Leiderer, and G. Schatz, Nat. Mater. 4, 203 (2005).

${ }^{15}$ D. Makarov, L. Baraban, I. L. Guhr, J. Boneberg, H. Schift, J. Gobrecht, G. Schatz, P. Leiderer, and M. Albrecht, Appl. Phys. Lett. 90, 093117 (2007).

${ }^{16}$ R. Streubel, D. Makarov, F. Kronast, V. Kravchuk, M. Albrecht, and O. G. Schmidt, Phys. Rev. B 85, 174429 (2012).

${ }^{17}$ R. Streubel, D. J. Thurmer, D. Makarov, F. Kronast, T. Kosub, V. Kravchuk, D. D. Sheka, Y. Gaididei, R. Schäfer, and O. G. Schmidt, Nano Lett. 12, 3961 (2012).

${ }^{18}$ R. Streubel, V. P. Kravchuk, D. D. Sheka, D. Makarov, F. Kronast, O. G. Schmidt, and Y. Gaididei, Appl. Phys. Lett. 101, 132419 (2012).

${ }^{19}$ T. C. Ulbrich, C. Bran, D. Makarov, O. Hellwig, J. D. Risner-Jamtgaard, D. Yaney, H. Rohrmann, V. Neu, and M. Albrecht, Phys. Rev. B 81, 054421 (2010).

${ }^{20}$ C. M. Günther, O. Hellwig, A. Menzel, B. Pfau, F. Radu, D. Makarov, M. Albrecht, A. Goncharov, T. Schrefl, W. F. Schlotter, R. Rick, J. Lüning, and S. Eisebitt, Phys. Rev. B 81, 064411 (2010).

${ }^{21}$ M. Albrecht and D. Makarov, Open Surf. Sci. J. 4, 42 (2012).

${ }^{22}$ M. V. Sapozhnikov, O. L. Ermolaeva, B. G. Gribkov, I. M. Nefedov, I. R. Karetnikova, S. A. Gusev, V. V. Rogov, B. B. Troitskii, and L. V. Khokhlova, Phys. Rev. B 85, 054402 (2012).

${ }^{23}$ D. Mitin, D. Nissen, P. Schädlich, S. S. P. K. Arekapudi, and M. Albrecht, J. Appl. Phys. 115, 063906 (2014).

${ }^{24}$ S. Thomas, D. Nissen, and M. Albrecht, Appl. Phys. Lett. 105, 022405 (2014).

${ }^{25}$ D. Nissen, D. Mitin, O. Klein, S. S. P. K. Arekapudi, S. Thomas, M.-Y. Im, P. Fischer, and M. Albrecht, Nanotechnology 26, 465706 (2015).

${ }^{26}$ R. Streubel, F. Kronast, U. K. Rößler, O. G. Schmidt, and D. Makarov, Phys. Rev. B 92, 104431 (2015).

${ }^{27}$ A. B. Butenko, A. A. Leonov, A. N. Bogdanov, and U. K. Rößler, Phys. Rev. B 80, 134410 (2009).

${ }^{28}$ I. E. Dzyaloshinskii, Sov. Phys. JETP 5, 1259 (1957).

${ }^{29}$ T. Moriya, Phys. Rev. 120, 91 (1960).

${ }^{30}$ M.-Y. Im, P. Fischer, K. Yamada, T. Sato, S. Kasai, Y. Nakatani, and T. Ono, Nat. Commun. 3, 983 (2012).

${ }^{31}$ R. P. Cowburn, D. K. Koltsov, A. O. Adeyeye, M. E. Welland, and D. M. Tricker, Phys. Rev. Lett. 83, 1042 (1999).

${ }^{32}$ K. Y. Guslienko, V. Novosad, Y. Otani, H. Shima, and K. Fukamichi, Phys. Rev. B 65, 024414 (2001). 